\title{
A FINITE PACKING PROBLEM
}

\author{
Norman Oler \\ (received March 13,1961)
}

The maximum density of packings of a given type into the whole of a Euclidean space is defined to be the limit of the maximum density of such packings into a cube as the edge of the cube goes to infinity.

For $E_{2}$, in particular, a number of well known results such as those due to A. Thue [1], L. Fejes-Toth [2], and C. A. Rogers [3] yield precise information about packings into the whole space. They are however of limited applicability to problems of finite packing in so far as each requires some restriction upon the boundary of the configuration.

In a paper which is shortly to appear [4] we obtain a result on the packings of plane convex disks which are free from such boundary restrictions. Our purpose in this note is to apply the result to a simple finite packing problem which may be stated:

Let $P_{1}, \ldots, P_{k}$ be a set of $k$ points contained in a closed region bounded by an equilateral triangle of side $n$ ( $n$ a positive integer). If the distance between any two of the points is at least 1 then it is conjectured that the maximum value of $k$ is the triangular number $\frac{1}{2}(n+1)(n+2) .{ }^{1}$

The conjecture can be affirmed by applying the following corollary to our theorem on the packing of convex disks.

Let $\pi$ be a Jordan polygon and $E$ a finite set of points which together satisfy the following conditions.

\footnotetext{
1 The problem was suggested to us in the course of conversation by Prof. Erdös while he was visiting McGill University.
}

Canad. Math. Bull. vol. 4, no. 2, May 1961. 
(i) The vertices of $\pi$ belong to $E$.

(ii) The set $E$ is contained in the closed region bounded by $\pi$.

(iii) The distance between any two points in $E$ is not less than 1.

Then the following inequality holds:

$$
\frac{2}{\sqrt{3}} \mathrm{~A}(\pi)+\frac{1}{2} \mathrm{M}(\pi)+1 \geq N
$$

where $A(\pi)$ is the area of the region bounded by $\pi, M(\pi)$ is the length of $\pi$ and $N$ is the number of points in $E$.

We consider a set $E$ contained in or on a triangle $T$ of sides $n$ ( $n$ a positive integer) with the property that the distance between any two points in $E$ is at least 1 . Let $H$ be the convex hull of $E$, i.e. the smallest convex set which contains $E$. It is clear that it is a polygon whose vertices are in $E$ and furthermore that it is contained in or coincides with $T$. Hence $A(H) \leq A(T)$. Further since $H$ is convex it is easy to prove that $M(H) \leq M(T)$.

The inequality (1) is applicable to the pair, $H$ and $E$, and yields

$$
\frac{2}{\sqrt{3}} \mathrm{~A}(\mathrm{H})+\frac{1}{2} \mathrm{M}(\mathrm{H})+1 \geq \mathrm{N} .
$$

Having

$$
A(H) \leq A(T)=\frac{\sqrt{3}}{4} n^{2}
$$

and

$$
M(H) \leq M(T)=3 n
$$

we find that

$$
N \leq 1+\frac{3}{2} n+\frac{1}{2} n^{2}=\frac{1}{2}(n+1)(n+2)
$$

It remains only to note that equality is realized for example if for $E$ we take that subset of the lattice generated by $(0,0)$, $(0,1)$ and $\left(\frac{1}{2}, \frac{\sqrt{3}}{2}\right)$ which is contained in or on the triangle $T$ whose vertices are $(0,0),(0, n)$ and $\left(\frac{1}{2} n, \frac{\sqrt{3}}{2} n\right)$.

We close with the remark that additional questions relating 
to the above and proposed in the same conversation remain open. Thus can one find a set of $\frac{1}{2}(n+1)(n+2)-1$ points, the distance between any two being at least one, in an equilateral triangle whose sides are $n-\varepsilon$ where $\varepsilon>0$ ? Though it is not difficult to construct such a set of $\frac{1}{2}(n+1)(n+2)-2$ points in a triangle of sides $n-\varepsilon$, this question remains open. One may also ask by how much a triangle of sides $n$ must be increased in order to contain such a set of $\frac{1}{2}(n+1)(n+2)+1$ points. In answer to this question our inequality provides but a weak estimate.

\section{REFERENCES}

1. A. Thue, Uber die dichteste Zusammenstellung von kongruenten Kreisen in einer Ebene, Christiania Vid. Selsk. $1(1910), 3-9$.

2. L. Fejes-Toth, Some packing and covering theorems, Acta Sci. Math. Szeged 12 (1950), 62-67.

3. C.A. Rogers, The closest packing of convex two dimensional domains, Acta Math. 86 (1951), 309-321.

4. N. Oler, An inequality in the geometry of numbers, Acta Math.,

\section{McGill University}

and

Columbia University 